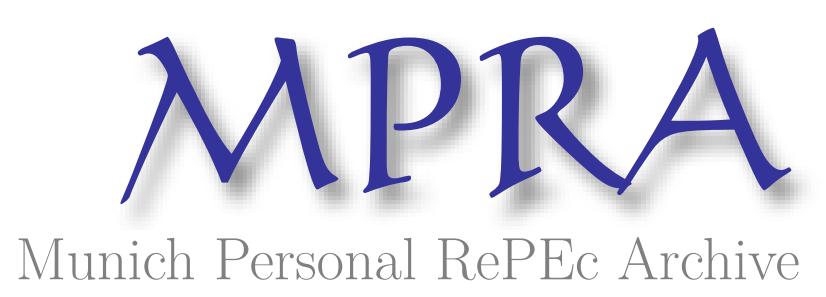

\title{
How Transport Costs Affect the Decision to Purchase a New or a Remanufactured Good
}

Batabyal, Amitrajeet and Beladi, Hamid

Rochester Institute of Technology, University of Texas at San Antonio

9 December 2017

Online at https://mpra.ub.uni-muenchen.de/85315/

MPRA Paper No. 85315, posted 20 Mar 2018 05:30 UTC 


\title{
How Transport Costs Affect the Decision to Purchase a New \\ or a Remanufactured Good ${ }^{1}$
}

by

\author{
Amitrajeet A. Batabyal ${ }^{2}$
}

and

Hamid Beladi ${ }^{3}$

1

For their helpful comments on two previous versions of this paper, we thank the Editor-in-Chief Yoshiro Higano, two anonymous reviewers, and audiences at several seminars. In addition, Batabyal acknowledges financial support from the Gosnell endowment at RIT. The usual absolution applies.

Department of Economics, Rochester Institute of Technology, 92 Lomb Memorial Drive, Rochester, NY 14623-5604, USA. Internet $\underline{\text { aabgsh@ rit.edu }}$

Department of Economics, University of Texas at San Antonio, One UTSA Circle, San Antonio, TX 78249-0631, USA. Internet Hamid.Beladi@utsa.edu 


\title{
How Transport Costs Affect the Decision to Purchase a New or a Remanufactured Good
}

\begin{abstract}
We provide the first strategic analysis of the interaction between a continuum of potentially green consumers and two firms in regional science. Firm 1 (2) sells new (remanufactured) toner cartridges. Each firm selects its price and a consumer purchases from the firm that offers her the highest utility. Utility is given by a surplus measure, the price, and by the transport cost incurred in traveling to a firm's location. We first derive the best response functions of the two firms. Second, we stipulate a numerical value for the surplus measure and show that when the two firms select their "monopoly" prices, the Nash equilibrium is unique. Third, we specify a linear transport cost function with a constant coefficient and show that the costlier it is for consumers to get to the locations of the two firms, the higher is the price charged by these two firms. Finally, our analysis shows that there is a need to study models in which the two toner cartridges are dissimilar, the interaction between consumers and firms is repeated, and behavioral factors are taken into account.
\end{abstract}

Keywords: Bertrand Model, New Good, Purchase, Remanufactured Good, Transport Cost JEL Codes: R41, Q57 


\section{Introduction}

Remanufacturing is the name given to an industrial process in which worn-out products are eventually restored to a like-new condition. As pointed out by Lund (1984) and Batabyal and Beladi (2016a, 2018), the remanufacturing process first involves the full disassembly of a discarded product. Next, usable parts are cleaned, refurbished, and then put into inventory. The product is then reassembled from the old parts---and occasionally with new parts as well---to manufacture a unit that is fully equivalent and sometimes superior in performance and expected lifetime to the original new product.

There is no gainsaying the fact that remanufacturing is now growing in popularity in many nations of the world. In this regard, it is worth noting that remanufacturing has become important in the United States mainly because of two reasons. First, inter alia, the Environmental Protection Agency (EPA) has implemented the so called "Comprehensive Procurement Guideline" in 1995. This guideline attempted to cut waste and promote resource conservation by ensuring that materials collected by recycling programs are utilized again to manufacture new products. ${ }^{4}$ Second, several firms have now seen actual cost savings as a result of their remanufacturing activities.

To see the source and the magnitude of the above mentioned actual cost savings, consider the following three examples from Mitra and Webster (2008). These researchers explain that in 1996, Ford was able to avoid the disposal of more than 67,700 pounds of toner cartridges and, as a result, saved \$180,000 in disposal costs. Second, between 1991 and 1997, Ford collected more than 332,000 pounds of toner cartridges and therefore was able to save $\$ 1.2$ million. Finally, in 1995, Union Carbide saved $\$ 75,000$ by sidestepping disposal costs. Given the growing

4

Go to https://vsc.gsa.gov/green/files/CPG.pdf for additional details. Accessed on 8 March 2018. 
significance of remanufacturing from both an environmental and a practical perspective, a burgeoning literature has now begun to analyze the usefulness of this industrial process from a variety of vantage points. We now briefly survey this literature.

A number of researchers have studied the process of remanufacturing from the standpoint of firms. Lebreton and Tuma (2006) survey the factors that are most likely to raise remanufacturing rates in their study of the disposal of 600,000 tons of used tires in Germany. Ferrer and Swaminathan (2006) analyze a multi-period model of competition between an original equipment manufacturer (OEM) and an independent operator (IO). They first demonstrate that the IO may intercept the cores of products made by the OEM to sell remanufactured products in future time periods. Next, they show that when the threat of competition rises, the OEM is more likely to fully utilize all available cores and offer the remanufactured product itself but at a lower price.

Might it be possible for remanufactured products to cannibalize new product sales? This important question has been studied by Atasu et al. (2010) who show that a product portfolio that includes both new and remanufactured products can make it likely for a firm to get to additional market segments and thereby block competition from low-end products or third-party manufacturers. Shi et al. (2015) examine the stability of the Nash equilibrium arising in the game between an OEM and a remanufacturer. They demonstrate that a higher willingness-to-pay (WTP) on the part of consumers can either reinforce or diminish the stability of the pertinent Nash equilibrium.

The competitive interaction between an OEM and a remanufacturer has recently been analyzed in a strategic or game-theoretic setting by Batabyal and Beladi (2016a). In the model utilized by these two researchers, the objective of both firms is to use product development 
expenditures to capture a dominant share of the market in which they are operating. Similarly, Batabyal and Beladi (2016b) also use a game-theoretic setting to show how consumers can pursue an environmental or green agenda versus a non-environmental or brown agenda through their product purchase decisions. Finally, Batabyal and Beladi (2018) study how the ability to advertise influences the market share enhancing strategies pursued by an OEM and a remanufacturer.

In the foregoing three paragraphs, we have discussed studies that have enhanced our understanding of the nature and the consequences of remanufacturing in a variety of different settings. This notwithstanding, our primary claim in this paper is that there are no studies in the existing regional science literature that have analyzed how spatial considerations influence the decision by consumers to purchase either a new or a remanufactured good. Second, we are also unaware of any studies in regional science that examine the ways in which transport costs incurred by potentially green or environmentally friendly consumers affects the decision to purchase either a new or a remanufactured good..$^{5}$

Given the lacuna in the literature that we have just mentioned, we adapt and apply Hotelling's (1929) well known price competition model to the problem of studying the strategic interaction between a continuum of potentially green consumers and two firms. The specific model we utilize is a static Bertrand model and, in game-theoretic parlance, we are analyzing a static game of complete information. ${ }^{6}$ Section 2 describes the model in which firm 1 sells new toner

\footnotetext{
5
}

Ferrer and Ayres (2000), Dethloff (2001), Madsen and Jensen-Butler (2004), Aghazadeh (2008) and Hamdouch et al. (2016) mention transport related issues in the context of remanufacturing, resource recovery, and sub-regional economic activity but the reader should note that there is no overlap between the topics covered in these five papers and the specific questions we address in the present paper.

See Tirole (1988, pp. 279-282) and Fudenberg and Tirole (1991, pp. 14-15) for textbook expositions of the Hotelling (1929) model. See Tadelis (2013, pp. 43-126) and Batabyal and Nijkamp (2016) for, respectively, a textbook account and a recent application of static games of complete information. The Hotelling (1929) model is a general model and hence either it or its variants have been used to study a variety of research topics in economics. Specifically, this model has been used to study topics as diverse as strategic outsourcing for supply chain management by Shy and Stenbacka (2003), the influences of store-brand introductions by Groznik and 
cartridges and firm 2 sells substantially similar but remanufactured toner cartridges. ${ }^{7}$ Each firm selects its own price and a consumer purchases a toner cartridge from the firm that offers her the highest utility. Utility is given by the difference between a surplus measure and the sum of the price and the transport cost borne by a consumer to travel to the relevant toner cartridge selling firm's location. Section 3 derives the best response functions of the two firms. Section 4 stipulates a numerical value for the surplus measure and then studies whether the resulting Nash equilibrium is unique. Section 5 introduces a linear transport cost function with a constant coefficient into the analysis and then solves for the Nash equilibrium. Section 6 introduces a linear transport cost function with a variable coefficient into the analysis and then demonstrates what happens to the ensuing Nash equilibrium when the variable coefficient becomes very small. Finally, section 7 concludes and then discusses three ways in which the research delineated in this paper might be extended in the future.

We contend that our paper makes progress in the regional science literature because it is the first to show how the Hotelling (1929) model, which is well known in the industrial organization literature, can be used to shed light on the ways in which transport costs affect the decision to purchase either a new or a remanufactured toner cartridge. More generally and moving beyond new and remanufactured toner cartridges, the way in which goods are shipped and the resulting transport costs can have an impact on the purchase decisions of environmentally conscious consumers for a whole host of goods including, but not limited to, fresh food and beverages. In

\footnotetext{
Heese (2010), facility location problems by Gastner (2011), and channel performance by Fu et al. (2013). It is important to understand that these four papers all contain new applications of the Hotelling model. As such, the results emanating from these papers are new from an applications perspective. Similarly, the results we obtain in our paper are also new in the sense that we use the Hotelling model to provide the first strategic analysis in regional science of the ways in which transport costs influence the decision to buy either a new or a remanufactured good.

For concreteness, in the remainder of this paper, we shall think of our remanufactured good as a toner cartridge. The reader should note that the model we analyze and the results we obtain are in no way dependent on the remanufactured good being a toner cartridge. Other real world examples of new and remanufactured goods include new and remanufactured cameras and new and remanufactured cell phones.
} 
other words, the purchase decisions of green consumers that are based at least in part on transport costs have implications for the environmental sustainability of alternate production processes and transport systems.

We now briefly summarize our main findings and then proceed to section 2 . First, for each of the two firms, the firm's own price is an increasing function of the price charged by its rival. Second, if the two toner cartridge producing firms select their "monopoly" prices (on which more in section 4) then this state of affairs leads to a unique Nash equilibrium of the game in which the participants are the consumers and the two firms. Third, the cheaper (costlier) it is for consumers to get to the locations of the two firms, the lower (higher) is the price charged by the two firms. Finally, the higher the transport cost of reaching one or the other firm, in the minds of the consumers, the greater is the differentiation between the two types of toner cartridges.

\section{The Theoretical Framework}

Consider a particular region in which there exists a continuum of potentially green or environmentally friendly consumers who are uniformly distributed on the line segment $[0,1]$. We work with the uniform distribution because of two reasons. First, as noted in Tirole (1988), this distribution is standardly used in modern game-theoretic discussions of price and non-price competition in the industrial organization literature. As such, our use of this distribution will make our analysis in this paper consistent with the extant literature in industrial organization in an important way. Second, the uniform distribution is also very convenient to work with.

Of the two firms in the model, firm 1 produces and sells new toner cartridges and firm 2 sells remanufactured toner cartridges. There is essentially no difference in the qualities of these two types of toner cartridges. With regard to the above mentioned line segment of unit length, firm 
1 is situated at point 0 and firm 2 is located at point 1 . The ith firm $(i=1,2)$ chooses its price $p_{i} \geq 0$ and each consumer purchases a toner cartridge from the firm that offers her the highest utility.

A consumer's utility $U$ is a function of a surplus measure $s$, the price $p_{i}$, and the distance $d_{i}$ that she has to travel to the relevant firm's location to make her purchase of either a new or a remanufactured toner cartridge. ${ }^{8}$ The reader should think of this $d_{i}$ as the transport cost associated with purchasing from firm $i$. In symbols, consumer $z \in[0,1]$ purchases a new toner cartridge (from firm 1) and not a remanufactured toner cartridge (from firm 2) if and only if

$$
U_{1}=s-p_{1}-d_{1}>U_{2}=s-p_{2}-d_{2},
$$

assuming, of course, that the utility from purchasing either the new or the remanufactured toner cartridge is positive. Note that in the game that we are analyzing, we have $d_{1}=z$ and $d_{2}=1-$ z. It is possible that a consumer's attitude toward a new toner cartridge is "behaviorally" different from her attitude to a remanufactured toner cartridge. A study of such behavioral economic factors is beyond the scope of this paper. What is salient in the present paper is that the new and the remanufactured toner cartridges are very similar to each other in terms of their quality in particular and their functionality more generally. Also, because our applied theoretic models are, in game-theoretic parlance, static games of complete information, consumers are aware of all the pertinent remanufacturing information and the provision of this information is assumed to be a legal requirement. Finally, note that we say a bit more about behavioral economic factors in the concluding section 7 of this paper.

Two aspects of our modeling thus far require some clarification. First, one can ask whether

8

The symbol $s$ denotes the surplus a consumer derives from consuming either the new or the remanufactured good. This surplus measure is gross of the price and the transport cost. It is common to include such a measure in models of the sort that we are analyzing in this paper. See Tirole (1988, p. 279) for additional details on this point. 
the surplus measures associated with the new and the remanufactured toner cartridges ought to be different. In other words, should we be working with $s_{1}$ and $s_{2}$ instead of a single $s$ ? In this regard, the references cited in the first paragraph of section 1 and the work of Agrawal et al. (2015) together tell us that the key purpose of remanufacturing is to give rise to products that are essentially like new products. In other words, any quality differences between these two types of products are minimal and, for all practical purposes, these two types of products have essentially the same quality. Because of this negligible quality difference, we do not work with different surplus measures for the new and the remanufactured toner cartridges and instead stick with the single surplus measure denoted by $s$.

Second, following Tirole (1988, pp. 96-97), when we think of the notion of a "product space," it is helpful to think in terms of vertically and horizontally differentiated product spaces. Since our modeling in this paper is based on Hotelling (1929), it should be clear to the reader that we are thinking of the two types of toner cartridges as being horizontally and not vertically differentiated. This means that the optimal choice of toner cartridge (new or remanufactured) depends on the particular consumer. This is entirely consistent with the "environmental story" we are telling in which there exists a continuum of possibly green or environmentally conscious consumers. The reader should also note that a remanufactured good is not the same as a used good. Specifically, relative to used goods, remanufactured goods typically have to pass much higher quality standards.

It should be clear to the reader that if the inequality in (1) were to be reversed then it would make more sense for consumer $z$ to purchase a remanufactured toner cartridge (from firm 2) and not a new toner cartridge (from firm 1). Finally, note that since a key objective of ours in this paper 
is to study the impact that transport costs have on consumer purchase decisions, in what follows, we shall abstract away from all other costs including the cost of producing either a new or a remanufactured toner cartridge. ${ }^{9}$ We now proceed to derive the best response functions of the two toner cartridge producing firms. ${ }^{10}$

\section{The Best Response Functions}

For the moment, let us assume that the surplus measure $s$ is sufficiently large so that all the consumers purchase either a new or a remanufactured toner cartridge. ${ }^{11}$ Using the language of modern industrial organization, the market for toner cartridges is covered. Next, consider the consumer $\tilde{z} \in[0,1]$ who is indifferent between purchasing either a new or a remanufactured toner cartridge. Note that consumer $\tilde{z}^{\prime} s$ distance from the new toner cartridge selling firm 1 is $\tilde{z}$ and that her distance from the remanufactured toner cartridge selling firm 2 is $1-\tilde{z} .{ }^{12}$ Therefore, this consumer's indifference tells us that the relationship

$$
s-p_{1}-\tilde{z}=s-p_{2}-(1-\tilde{z})
$$

holds. ${ }^{13}$ Equation (2) allows us to solve explicitly for this indifferent consumer $\tilde{z}$. We get

$$
\tilde{z}=\frac{1-p_{1}+p_{2}}{2} \text {. }
$$

\section{9}

If the cost of producing either a new or a remanufactured toner cartridge can be modeled with a linear cost function then it would be straightforward to account for this cost in the analysis and the basic structure of our subsequent mathematical results in sections 4 through 6 below would be unchanged.

10

In order to make this paper interesting to as many readers as possible, we have decided to first derive the best response functions and to then use these functions to solve for the Nash equilibrium in section 4 . This is standard practice in many game theory textbooks. See Tadelis (2013, pp. 84-95) for some examples. Having said this, we recognize that we could also first state the Nash equilibrium in the form of a Proposition and then proceed to provide a proof of this Proposition.

In section 4 below, we study the changes that arise in the market for new and remanufactured toner cartridges when this assumption is violated.

12

Note the use of the assumption that our environmentally conscious consumers are uniformly distributed on the interval [0,1]. 13

Note that the right-hand-side (RHS) and the left-hand-side (LHS) of equation (2) are greater than zero. 
Using equation (3), we can articulate the demand functions confronting the new and the remanufactured toner cartridge selling firms as a function of the two prices $p_{1}$ and $p_{2}$. We get

$$
q_{1}\left(p_{1}, p_{2}\right)=\tilde{z}=\frac{1-p_{1}+p_{2}}{2}
$$

and

$$
q_{2}\left(p_{1}, p_{2}\right)=1-\tilde{z}=\frac{1+p_{1}-p_{2}}{2}
$$

Using equation (4), the new toner cartridge producing firm 1's profit maximization problem is

$$
\max _{p_{1}}\left\{\frac{1-p_{1}+p_{2}}{2}\right\} p_{1}
$$

The first order necessary condition for an optimum is $1-2 p_{1}+p_{2}=0$ and this condition tells us that the new toner cartridge producing firm 1's best response function is

$$
p_{1}=\frac{1+p_{2}}{2}
$$

Because of the symmetry between the two firms in our model, ${ }^{14}$ we deduce that the

14

In section 2, we provided a detailed explanation of the reasons for working with a single surplus measure $s$ and for only considering a horizontally differentiated product space. The reader should note that these two features together also provide the 
remanufactured toner cartridge producing firm 2's best response function is

$$
p_{2}=\frac{1+p_{1}}{2}
$$

Inspecting equations (7) and (8), we see that for each of the two firms, the firm's own price is an increasing function of the price charged by its rival. In game-theoretic parlance, the new and the remanufactured goods are strategic complements in prices. We now first stipulate a numerical value for the surplus measure $s$ and then study the properties of the Nash equilibrium in the game between the consumers and the new and the remanufactured toner cartridge producing firms.

\section{A Specific Surplus Value and the Nash Equilibrium}

If we solve equations (7) and (8) simultaneously, then it is straightforward to see that in the resulting Nash equilibrium we have $p_{1}=p_{2}=1$ and that this equilibrium is unique. In addition, substituting $p_{1}=p_{2}=1$ in equation (3) tells us that $\tilde{z}=1 / 2$. In words, this last result tells us that exactly one-half of all consumers purchase the new toner cartridge and the remaining half purchases the remanufactured toner cartridge.

Now, suppose that the surplus measure $s=1$ in the remainder of this paper. Why are we studying this particular case? To see why, recall the assumption we made in the first paragraph of section 3 about $s$ being sufficiently large so that all the consumers purchase either a new or a remanufactured toner cartridge. We now depart from the extant literature and study what happens in our market for toner cartridges when this assumption does not hold. Having said this, note that there are a very large number of values of the surplus measure $s$ that correspond to the case where $s$ is not sufficiently large. Therefore, rather than focus on a very large number of cases, we wish to 
illustrate the basics of how the analysis changes when $s$ is not sufficiently large. In our case, computations show that this can effectively be done by studying any value of $s<1.5$. Now 1 is an easily recognized positive integer and, in addition, $s=1$ satisfies the preceding constraint. This is why we are studying the $s=1$ case.

Straightforward substitution shows that the utility of the consumer who is indifferent between purchasing a new and a remanufactured toner cartridge $(\tilde{z}=1 / 2)$ is $s-p_{1}-$ $1 / 2=-1 / 2$ which is obviously negative and hence this consumer will not purchase a toner cartridge. ${ }^{15}$ Because the consumers we are studying constitute a continuum in the interval $[0,1]$, a continuity argument tells us that an interval of consumers around consumer $\tilde{z}$ will also not purchase a toner cartridge. This means that when $s=1$, the best response functions we have computed in section 3 and our analysis thus far are invalid.

Given this state of affairs, what is the next logical step that we might take? We contend that the next logical step is to consider the monopoly case to see how each of the two toner cartridge producing firms would price its product on the assumption that the other firm does not exist. Put differently, we are temporarily supposing that there is only a single toner cartridge producing firm that has effectively monopolized the market. Suppose also that this producer is firm 1 . Then, the monopolist firm 1's profit maximization problem is

$$
\max _{p_{1}}\left\{\left(1-p_{1}\right) p_{1}\right\}
$$

where the first term in parentheses inside the curly brackets denotes the demand confronting this firm and the second term is the price it charges. The solution to the above problem is $p_{1}=1 / 2$. This means that all consumers who lie in the interval $z \in[0,1 / 2]$ will purchase a new toner 15

We need $s \geq 1.5$ for consumer $\tilde{z}=1 / 2$ to be indifferent between purchasing and not purchasing when $p_{1}=p_{2}=1$. In this instance, all the other consumers will prefer purchasing a toner cartridge to not purchasing one. 
cartridge from firm 1 and no other consumers will purchase a toner cartridge. Now, because the two firms in our model are symmetrical, if the remanufactured toner cartridge producing firm 2 were the monopolistic firm then its optimal price would be $p_{2}=1 / 2$. In addition, all consumers in the interval $z \in[1 / 2,1]$ would purchase only a remanufactured toner cartridge from firm 2 and no other consumers would buy a toner cartridge. Finally, observe that when $s=1$, the result in section 3 that $p_{1}=p_{2}=1$ no longer holds.

The upshot of the discussion in the preceding paragraph is that if the two toner cartridge producing firms select their monopoly prices $p_{1}=p_{2}=1 / 2$ then this is, in fact, the unique Nash equilibrium of the game involving the consumers and the two toner cartridge producing firms. Further, because of the geographical locations of the two firms---firm 1 at point 0 and firm 2 at point 1 in the $[0,1]$ line segment---each firm effectively covers one-half of the market. So, the two toner cartridge producing firms solve static maximization problems and, together, they cover the market for toner cartridges. This equilibrium is very different from the section 3 equilibrium because the surplus measure $s$ is assumed to not be sufficiently large but instead to equal unity in this section. How does the analysis in this section and the Nash equilibrium in particular change when we have a linear transport cost function with a constant coefficient? We now proceed to answer this question.

\section{Linear Transport Costs with a Constant Coefficient}

Suppose that the transport cost borne by the consumers to travel to the location of the ith firm is given by $1 / 2 d_{i}$ where the constant coefficient is clearly $1 / 2$. This means that a consumer will purchase a new and not a remanufactured toner cartridge if and only if $s-p_{1}-(1 / 2) d_{1}>$ $s-p_{2}-(1 / 2) d_{2}$. To express the best response functions of the two toner cartridge producing 
firms, let us begin by recalling the consumer $\tilde{z} \in[0,1]$ who is indifferent between purchasing the two kinds of toner cartridges that are available for sale. We know that this consumer's distance from firm 1 is $\tilde{z}$ and that her distance from firm 2 is $1-\tilde{z}$. As in section 3 , this consumer's indifference between the two kinds of available toner cartridges tells us that we must have

$$
s-p_{1}-(1 / 2) \tilde{z}=s-p_{2}-(1 / 2)(1-\tilde{z})
$$

Solving equation (10) for $\tilde{z}$, we get

$$
\tilde{z}=\frac{1}{2}-p_{1}+p_{2}
$$

For given prices $p_{1}$ and $p_{2}$, we follow the reasoning employed in section 3 to express the demand functions facing the two toner cartridge producing firms. We get

$$
q_{1}\left(p_{1}, p_{2}\right)=\tilde{z}=\frac{1}{2}-p_{1}+p_{2}
$$

and

$$
q_{2}\left(p_{1}, p_{2}\right)=1-\tilde{z}=\frac{1}{2}+p_{1}-p_{2}
$$

Using equation (12), the new toner cartridge producing firm 1's profit maximization problem is 


$$
\max _{p_{1}}\left\{\frac{1}{2}-p_{1}+p_{2}\right\} p_{1}
$$

The first order necessary condition for an optimum is $1 / 2-2 p_{1}+p_{2}=0$ and this condition tells us that firm 1's best response function is

$$
p_{1}=\frac{1+2 p_{2}}{4}
$$

By symmetry, the remanufactured toner cartridge producing firm 2's best response function is

$$
p_{2}=\frac{1+2 p_{1}}{4}
$$

Solving equations (15) and (16) simultaneously, we get $p_{1}=p_{2}=1 / 2$. Substituting this last finding in equation (11) tells us that $\tilde{z}=1 / 2$. Note the dependence of the optimal prices on the transport cost borne by the consumers to reach the locations of the two toner cartridge producing firms. In particular, we see that the optimal prices charged by the two firms equal one-half and that this also equals the constant marginal transport cost coefficient. Intuitively, this tells us that, ceteris paribus, the cheaper (costlier) it is for consumers to get to the locations of the two firms selling the two different kinds of toner cartridges, the lower (higher) will be the price charged by the two firms for these same cartridges.

We now claim that the triple $\left(p_{1}, p_{2}, \tilde{z}\right)=(1 / 2,1 / 2,1 / 2)$ is the Nash equilibrium of the game between the consumers and the new and the remanufactured toner cartridge producing firms. To confirm this claim, let us focus on the indifferent consumer $\tilde{z} \in[0,1]$. We have to show that in 
the claimed Nash equilibrium, the utility to this consumer from purchasing a new toner cartridge from firm 1 is zero. Making the relevant substitutions, we see that this consumer's utility is $s-p_{1}-\tilde{z}=1-(1 / 2)-(1 / 2)=0$. This result tells us that consumer $\tilde{z}$ is indeed indifferent between purchasing and not purchasing a toner cartridge and hence every other consumer prefers to purchase rather than not purchase a toner cartridge. Our final task in this paper is to study the impact that a linear transport cost function with a variable coefficient has on the interaction between the two firms that seek to sell new and remanufactured toner cartridges to the continuum of consumers in the region under study.

\section{Linear Transport Costs with a Variable Coefficient}

The transport cost borne by the consumers to travel to the locations of the two toner cartridge selling firms is now given by $\tau d_{i}$ where $\tau \in[0,1 / 2]$. We now provide a three-part justification for our choice of this particular linear transport cost function. First, a perusal of either Tirole (1988, p. 279) or Carlton and Perloff (1994, p. 304) shows that in the modern industrial organization literature, it is quite standard to use linear transport cost functions when studying price competition models of the sort that we are analyzing in this paper. Second, to be consistent with the analysis in section 5 which uses a fixed coefficient linear transport cost function, we have chosen to focus on a linear transport cost function in this section as well. Finally, our choice of the transport cost coefficient $\tau$ is governed by the fact that we want to study the limiting behavior of the Nash equilibrium in the interaction between the two firms and the consumers when this coefficient $\tau$ approaches zero. We agree that our subsequent mathematical results in this section are contingent upon our choice of the $\tau$ coefficient. We also agree that working with a linear transport cost function is less general than working with a transport cost function with no particular 
functional form. However, the widespread use of linear transport cost functions and our desire to obtain analytical results leads us to use the specific formulation that we do use.

Following the analysis in section 5, let us, once again, begin the analysis by focusing on the indifferent consumer $\tilde{z}$. From this consumer's indifference, we know that we must have

$$
s-p_{1}-\tau \tilde{z}=s-p_{2}-\tau(1-\tilde{z}) .
$$

Solving equation (17) for $\tilde{z}$, we get

$$
\tilde{z}=\frac{1}{2}+\frac{p_{2}-p_{1}}{2 \tau} .
$$

Now, given our assumptions thus far and the two prices $p_{1}$ and $p_{2}$, the demand functions confronting the two toner cartridge selling firms are

$$
q_{1}\left(p_{1}, p_{2}\right)=\tilde{z}=\frac{1}{2}+\frac{p_{2}-p_{1}}{2 \tau},
$$

and

$$
q_{2}\left(p_{1}, p_{2}\right)=1-\tilde{z}=\frac{1}{2}+\frac{p_{1}-p_{2}}{2 \tau} .
$$

Using equation (19), the new toner cartridge producing firm 1's profit maximization problem is 


$$
\max _{p_{1}}\left\{\frac{1}{2}+\frac{p_{2}-p_{1}}{2 \tau}\right\} p_{1}
$$

The first order necessary condition for an optimum is $(1 / 2)+\left(p_{2}-2 p_{1}\right) / 2 \tau=0$ and this condition can be solved to give us firm 1's best response function. We get

$$
p_{1}=\frac{p_{2}+\tau}{2}
$$

By symmetry, we infer that the best response function for the remanufactured toner cartridge producing firm 2 is

$$
p_{2}=\frac{p_{1}+\tau}{2}
$$

Solving the two best response functions in (22) and (23) simultaneously, we get $p_{1}=p_{2}=$ $\tau$. Substituting this value for the two prices in equation (18), we get $\tilde{z}=1 / 2$. Now, from the section 5 analysis, we deduce that for any $\tau \in[0,1 / 2]$, consumer $\tilde{z}$ will prefer to purchase a toner cartridge rather than not purchase any and that this is also the purchase decision that will be made by all the other consumers.

To see the impact of the transport cost $\tau d_{i}$ on the prices charged by the two toner cartridge selling firms, observe that we can now generalize the conclusions obtained for the constant marginal transport cost coefficient case in section 5. In this regard, note first that the optimal prices charged by the two firms equal $\tau$ which is the variable marginal transport cost coefficient. Intuitively, the specific impact of $\tau$ is as follows. When $\tau$ increases (decreases), the optimal 
prices charged by the two firms also increase (decrease). Looked at a little differently, the marginal transport cost $\tau$ causes the two toner cartridge selling firms to be differentiated. In turn, this differentiation implies that when the marginal transport cost borne by consumers is high, the optimal prices that are charged are high and competition between the two firms for the same consumers is attenuated. In contrast, when the marginal transport cost is low, the optimal prices are low and competition between the two firms is intense. In the limit as $\tau$ approaches zero, the prices charged by the two firms also approach zero and this can be thought of as the most extreme form of price competition between the two toner cartridge selling firms.

We recognize that the marginal transport $\operatorname{cost} \tau$ in this section corresponds to the fixed marginal transport cost $1 / 2$ in section 5 . This notwithstanding, we contend that it is not helpful to think of section 5 as a "special case" of this section because of three reasons. First and most generally, the analysis in this paper is cumulative in the sense that each section builds on the analysis conducted in the preceding section. So, in this way of looking at our paper, there would be no meaningful section 6 without a section 5 first. Second and more specifically, sections 5 and 6 are complementary in the sense that the discussion in section 6 refers to and builds on the section 5 analysis on more than one occasion. Finally, sections 5 and 6 focus on and answer different questions. Whereas section 5 is concerned primarily with determining the Nash equilibrium of the game involving the consumers and the two toner cartridge producing firms, section 6 is mainly concerned with explicating the intensity of the price competition between the two toner cartridge producing firms in a limiting case. This completes our theoretical discussion of the ways in which transport costs influence a consumer's decision to purchase either a new or a remanufactured toner cartridge. 


\section{Conclusions}

In this paper, we analyzed a Bertrand model of the interaction between a continuum of potentially green consumers and two toner cartridge selling firms. Our main findings are as follows. First, for each of the two firms, the firm's own price is a rising function of the price charged by its rival. Second, if the two toner cartridge producing firms select their "monopoly" prices then this situation leads to a unique Nash equilibrium of the game in which the participants are the consumers and the two firms. Third, the cheaper (costlier) it is for consumers to reach the locations of the two firms, the lower (higher) is the price charged by the two firms. Finally, the higher the transport cost of getting to one or the other firm, in the minds of the consumers, the greater is the differentiation between the two types of toner cartridges.

What new research "puzzles" does our analysis in this paper give rise to? To answer this question, let us briefly consider three distinct "puzzles." A puzzle is that we do not know the connection between transport costs and consumer purchase decisions when the divergent costs of producing, say two goods, are explicitly modeled. A second puzzle is that we do not know what results we could obtain by analyzing a model of the sort studied in this paper but where the interaction between the potentially green consumers and the two toner cartridge producing firms is repeated over time. A third and final puzzle is that we are unaware of the impacts that behavioral economic factors---such as dissimilar attitudes toward new and remanufactured toner cartridges---would have on the decision to purchase either a new or a remanufactured good in the presence of transport costs. Future studies that shed light on these three puzzles will provide valuable insights into the nexuses between transport costs, the production of new and remanufactured goods, and environmental protection. 


\section{References}

Aghazadeh, S. 2008. The success of reverse logistics in supporting the environment: The case of the computer industry, International Journal of Environment and Sustainable Development, 7, 452-464.

Agrawal, V., Atasu, A., and Van Ittersum, K. 2015. Remanufacturing, third-party competition, and consumers' perceived value of new products, Management Science, 61, 60-72.

Atasu, A., Guide, V.D.R., and Van Wassenhove, L.N. 2010. So what if remanufacturing cannibalizes my new product sales? California Management Review, 52, 56-76.

Batabyal, A.A., and Beladi, H. 2016a. A game model of competition for market share between a new good producer and a remanufacturer, Economics Bulletin, 36, 963-969.

Batabyal, A.A., and Beladi, H. 2016b. A game model of new and remanufactured goods, brown and green consumers, and market share dominance, Journal of Quantitative Economics, $14,345-354$.

Batabyal, A.A., and Beladi, H. 2018. Advertising and competition for market share between a new good producer and a remanufacturer, German Economic Review, 19, 113-118.

Batabyal, A.A., and Nijkamp, P. 2016. A game model of international trade, transport costs, invasive species, and protocol compliance, Transportation Research Part D, 46, 267-272.

Carlton, D.W., and Perloff, J.M. 1994. Modern Industrial Organization, $2^{\text {nd }}$ edition. Harper Collins College Publishers, New York, NY.

Dethloff, J. 2001. Vehicle routing and reverse logistics: The vehicle routing problem with simultaneous delivery and pick-up, OR Spektrum, 23, 79-96.

Ferrer, G., and Ayres, R.U. 2000. The impact of remanufacturing in the economy, Ecological 
Economics, 32, 413-429.

Ferrer, G., and Swaminathan, J.M. 2006. Managing new and remanufactured products, Management Science, 52, 15-26.

Fu, Y., Lai, K.K., and Lian, L. 2013. Bricks or clicks: The impact of manufacturer's encroachment on both manufacture-owned and traditional retail channels, Asia Pacific Journal of Marketing and Logistics, 25, 695-714.

Fudenberg, D., and Tirole, J. 1991. Game Theory. MIT Press, Cambridge, MA.

Gastner, M.T. 2011. Scaling and entropy in p-median facility location along a line, Physical Review, E84, 1-7.

Groznik, A., and Heese, H.S. 2010. Supply chain interactions due to store-brand interactions: The impact of retail competition, European Journal of Operational Research, 203, 575-582.

Hamdouch, Y., Qiang, Q.P., and Ghoudi, K. 2016. A closed-loop supply chain equilibrium model with random and price-sensitive demand and return. Forthcoming, Networks and Spatial Economics.

Hotelling, H. 1929. Stability in competition, Economic Journal, 39, 41-57.

Lebreton, B., and Tuma, A. 2006. A Quantitative approach to assessing the profitability of car and truck tire remanufacturing, International Journal of Production Economics, 104, 639-652.

Lund, R.T. 1984. Remanufacturing, Technology Review, 87, 19-23, 28-29.

Madsen, B., and Jensen-Butler, C. 2004. Theoretical and operational issues in sub-regional economic modelling, illustrated through the development and application of the LINE model, Economic Modelling, 21, 471-508.

Mitra, S., and Webster, S. 2008. Competition in remanufacturing and the effects of government 
subsidies, International Journal of Production Economics, 111, 287-298.

Shi, L., Sheng, Z., and Xu, F. 2015. The dynamics of competition in remanufacturing: A stability analysis, Economic Modelling, 50, 245-253.

Shy, O., and Stenbacka, R. 2003. Strategic outsourcing, Journal of Economic Behavior and Organization, 50, 203-224.

Tadelis, S. 2013. Game Theory: An Introduction. Princeton University Press, Princeton, NJ.

Tirole, J. 1988. The Theory of Industrial Organization. MIT Press, Cambridge, MA. 